Uluslararası Mühendislik

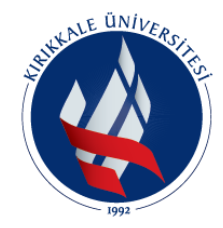

UMAGD, (2020) 12(2), 579-586.

International Journal of

Engineering Research and

Development

UMAGD, (2020) $12(2), 579-586$.

10.29137/umagd.747680

Cilt/Volume:12 Sayı/Issue:2 Haziran/June 2020

Araştırma Makalesi / Research Article

\title{
Non-Contact Vibration Detection Software and Hardware Design with Image Processing Method to Reveal Substantial Changes in Structures and Systems
}

\author{
Emre Metin $^{1}$ iD, Özgür Karagülle ${ }^{1}$ iD, Abdullah Kayalak ${ }^{\text {iD }}$, Mustafa Karagülle ${ }^{\text {iD }}$,Ertuğrul Çam *2 iD

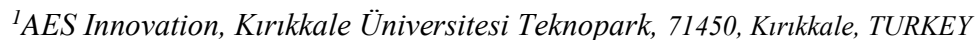 \\ ${ }^{2}$ Kirlkkale University, Department of Electrical \& Electronics Engineering, 71450, Kirlkkale, TURKEY
}

Başvuru/Received: 03/05/2020

Kabul / Accepted: 17/06/2020

Çevrimiçi Basım / Published Online: 30/06/2020

Son Versiyon/Final Version: 30/06/2020

\begin{abstract}
Many types of equipment are used for vibration detection in buildings and systems. However, these systems have their own problems. In this article, a combined software and hardware system is designed for the solution of these problems. The system has been designed with the Motion Magnification Analysis (MMA) method, which stands out in the literature studies. C \# language and .NET environment have been used as software. The Accord library was used for the image processing process. These libraries were also used for the analysis of subspaces and components of the image. During the study, three new algorithms that have not been applied in the past and not in the literature have been developed. The first of these is the derivative of the method known as Color Magnification, which analyzes the color space. The second is the derivative of the Motion Magnification method, which magnifies the vibration to the visible level. And the third one is the Frequency Determination algorithm, which enables frequency measurement. With this solution, it is provided to make an analysis of the images taken with devices with relatively slow cameras such as mobile phones/tablets as well as fast cameras. All results were compared with a touchable vibration analyzer for benchmarking and showed that the proposed method was successful.
\end{abstract}

Key Words

"vibration, noncontact, motion magnification analysis, colour magnification" 


\section{Introduction}

Today, most of the machines used in the industry do business with a rotary motion. In order for these machines to perform their duties without interruption, the malfunctions that will occur must be determined at the initial stage and the malfunction must be eliminated by performing maintenance. Vibration analysis is one of the most appropriate methods for this job. If the vibrations caused by the malfunctions of rotating machines are well understood, it is easy to identify the malfunctions. Increased gap between the parts working on the machines, abrasion, crack formation, and similar factors cause vibration. The most important factor that causes them is the internal and external forces acting on the machine. Therefore, it is desirable that these forces be as small as possible when designing machines. Even a properly manufactured machine generates a certain level of vibration during operation. This is a situation that cannot be avoided in practice. The important thing is to keep this vibration level under control to remain at an acceptable level. Just like when an area in the human body is disturbed there is an increase in the level of vibration when a malfunction occurs in the machine. Using this feature, the relationship between malfunctions occurring in rotating machines and vibration has been determined.

Since malfunctions and vibration relations are detected in rotating machines, vibration analysis has been used while planning maintenance activities in factories. To obtain healthy and accurate results from vibration analysis is possible with the knowledge and experience gained in this field. The combination of high exactness accelerometers and the capacity to process the Fourier change with the FFT calculation has been permitted the advancement of quick apparatuses that can analyze the real condition of the machines (Correa \& Guzman, 2020). Nowadays, vibration sensors were applied to a few applications because of their expenses and the requirement for master staff. These underlying ideas are supplemented by other new advances, for example, ultrasonic, thermography, acoustic sensors, and omnidirectional mouthpieces.

Condition checking frameworks are basic for understanding the conduct of machines, particularly pivoting machines. These are key components to foresee mistakes in the majority of the things that join machines (Chen et al., 2015). They can decide the kind of deficiency and the second when an imperfection shows up in a specific part. They likewise give enough information to improve the machine plan, which can be investigated to relate outstanding tasks at hand, lifecycle, and sort of shortcoming. This information is a significant contribution to future structures. The structure and meaning of a condition checking framework characterize the sort and measure of information to be delivered after the machine is set up and worked.

Video amplification is a strategy that works by expanding shading and brilliance varieties in singular pixels. It is in this way perfect for following changes in shading, however it can likewise be utilized to uncover inconspicuous sub-pixel movement, giving more consideration to preparing distinctive spatial frequency varieties. The picture is first isolated into spatial recurrence parts, that is, various segments relying upon the quality and sharpness of an edge or other picture attributes (Gonzales, 1987). The subsequent advance applies pixel-based fleeting amplification lastly, the parts are reassembled with weighting as indicated by a predefined enhancement factor to expand the movement. Utilizing video amplification causes little, sub-pixel relocations related to vibration to be obvious.

At the point when the movement shows up in the video, fixed focuses can be followed with the goal that the extent of the vibration can be acquired. It is noted that similarly as with any discrete-time framework, the example recurrence must be sufficiently high to catch the ideal sign as indicated by the Nyquist rule. Thusly, in this investigation, it has been utilized a fast camera to screen the vibrations happening in the sound recurrence go. (Davis et al., 2014) utilized the blinds of a standard buyer camera to deliver perceptible sound from the video not considered here.

In this paper, it has been evaluated traditional vibration monitoring methods and developing a new approach for condition monitoring systems with contactless video processing and analysis methods.

\section{Basics of Vibration}

The premise of most molding checking frameworks is the investigation of vibration signals, so a fundamental piece of understanding the circumstances and end results of vibrations is the investigation of wavering developments and connections between various pieces of a machine, structure, or arrangement of machines. These wavering developments are known as mechanical vibrations or, all the more for the most part, vibrations with regards to plan and machine and auxiliary investigation. The quintessence of molding checking is the investigation of the connection between the information signal (wellspring of vibration) and the yield reaction (yield signal) and the advancement of the machine's dynamic conduct. In different cases, in spite of the fact that there are some extraordinary cases to be examined in different segments, machines can be considered as a straight framework and the yield sign will have a direct reaction to the excitation powers. While a machine is an intricate arrangement of numerous mechanical components, its dynamic reaction can be spoken to as a straightforward mass framework.

So as to dissect the vibrational occasions, it is important to speak to the developments so the attributes of the vibrations can be characterized as deterministic or irregular. The least complex portrayal of a machine is made by romanticizing vibration developments 
as an upheld mass of movement. With a spring and a clingy damper (Fig. 1.1). The mass is viewed as a solitary molecule, and the bend is a component that accommodates Hook's law, the firmness steady of $\mathrm{k}$, the mass of the molecule $\mathrm{m}$, and the gooey consistent $\mathrm{c}$, which is the damping of this development. This spring oscillation's force is given by $\mathrm{F}_{\mathrm{r}}=\mathrm{kx}$, where $\mathrm{x}$ is the displacement that the mass $m$ suffers. Also the damper's force is given by $\mathrm{F}_{\mathrm{a}}=\mathrm{cv}$, where $\mathrm{c}$ is the damping coefficient and $\mathrm{v}$ is the speed of the mass motion $m$. External forces applied to the mechanical system created by mass, spring and shock absorber, F(t) (Correa \& Guzman, 2020).

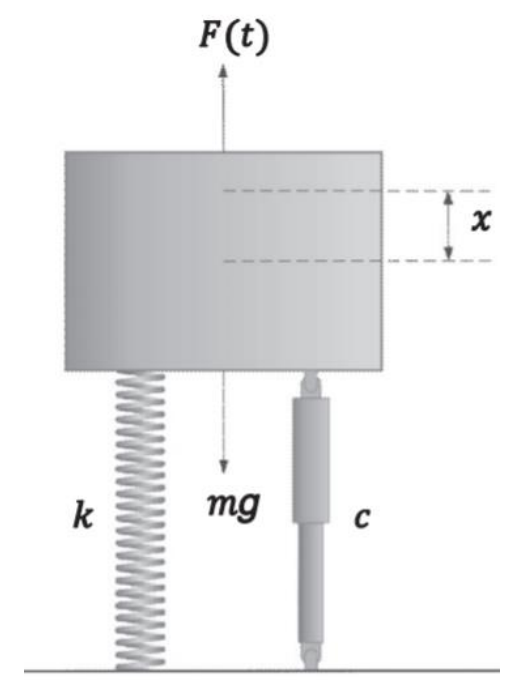

Fig. 1. Model of a vibratory system.

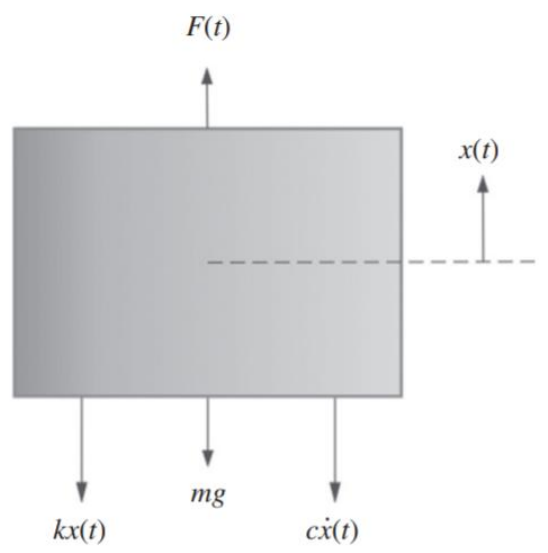

Fig. 2. The acting forces on a vibratory system.

The equation of motion is obtained as,

$-k x-c v+F(t)-m g+F_{e s t}=m a$

in which Fest is the mass weight force, g, acceleration of gravity and a is the acceleration of the System. This formula simplified in such a way as:

$\mathrm{ma}+\mathrm{kx}+\mathrm{cv}=\mathrm{F}(\mathrm{t})$

Considering the notation as follows

$$
\begin{aligned}
& v=\lim (\Delta x / \Delta t) \Delta t \rightarrow 0 ; \frac{d x}{d t}=\dot{x} \\
& a=\lim (\Delta v / \Delta t) \Delta t \rightarrow 0 ; d v / d t=d^{2} / d t^{2}=\ddot{x}
\end{aligned}
$$


The motion equation can be given as,

$\mathrm{m} \dot{x}+\mathrm{c} \ddot{x}+\mathrm{kx}=\mathrm{F}(\mathrm{t})$

This recipe is the condition of the movements of a level of opportunity with constrained vibrations and damping. The idea of "level of opportunity" alludes to the base record of directions required to characterize the kinematics of a repairman component. Along these lines, the framework has a level of opportunity, since just the uprooting x must be known to decide the situation of the mass m.

The arrangement of the second-request differential condition has two components, the homogenous arrangement and the specific arrangement. The homogenous arrangement speaks to the free vibration development and the specific arrangement relates to the constrained vibration of the mass. The portrayal of the oscillatory development of the mass $\mathrm{m}$ is gotten by characterizing the condition of development as per the recurrence (f) and the sufficiency (x).

The swaying recurrence is the quantity of unit times the development rehashes, and its abundancy is the extent of the greatest relocation. From the previous, it can be inferred that $\mathrm{f}=\mathrm{n} / \mathrm{t}$, where $\mathrm{n}$ is the number of cycles completed in an interval $\mathrm{t}$. If $\mathrm{n}=1$, it can be said that $\mathrm{t}=\mathrm{T}$ is the oscillation period. Since a harmonic oscillation repeats regularly, it can be shown in Fig. 1.3, where it can be seen that for a cycle to be complete, the period must be $\mathrm{T}=2 \pi / \omega$. In the equation, $\omega$ is the angular frequency, where $\omega=2 \pi \mathrm{f}$. Considering initial conditions, the phase angle can be defined as $\phi=\tan -1(\mathrm{v} 0 / \mathrm{x} 0 \omega)$. Fig. 1.4 Illustrates the perspectives that characterize the pinnacle sufficiency, the top to top, the normal worth, and the root mean square (RMS) in an intermittent sign and an arbitrary sign (without clear request). Top-to-top worth shows absolute dislodging of vibration from start to finish. This worth is important, for instance, when given most extreme power, greatest abundancy in a machine part. The pinnacle esteem is valuable for demonstrating transient impacts, paying little heed to the historical backdrop of the vibration. At the point when it is important to know the normal estimation of the vibration, the accompanying proclamation is utilized:
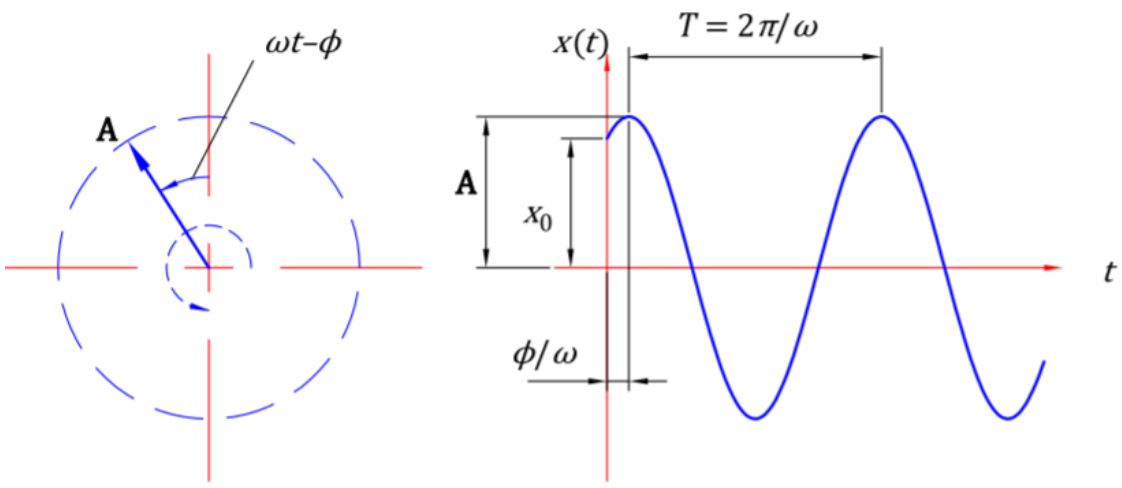

Fig. 3. Representation of a harmonic oscillation.

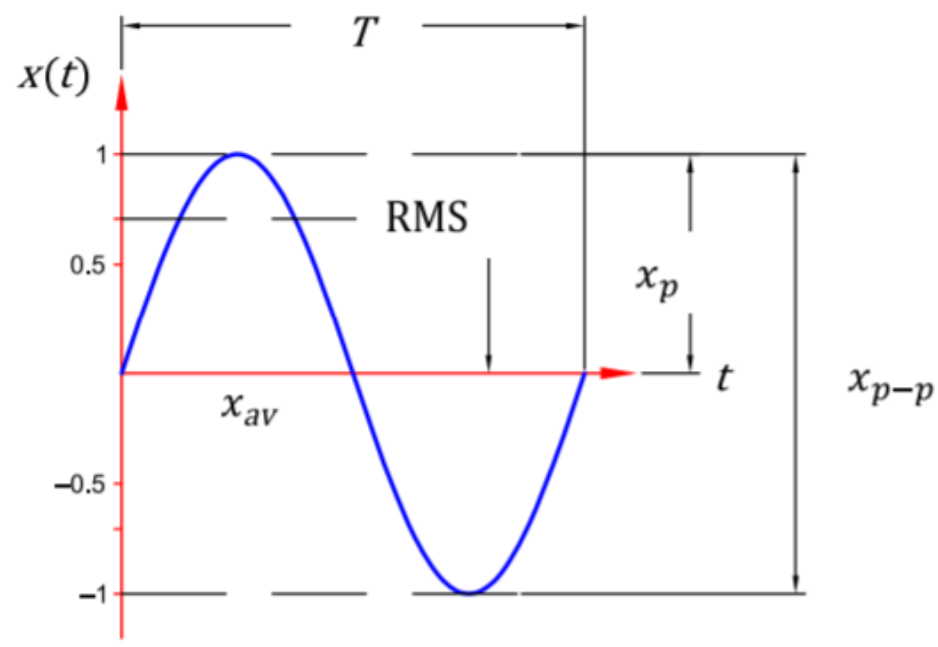

Fig. 4. Definition of the parameters of a vibration signal.

$x_{\text {prom }}=1 / \mathrm{T} \int_{0}^{T} x d t$ 
The normal of a sinusoidal capacity is consistently zero; along these lines, it's worth has a negligible commitment to the investigation of a vibration signal and won't work for a molding checking framework. There is another estimation of the mean plentifulness of the sinusoidal sign, the root mean square (RMS), given by the accompanying articulation:

$\mathrm{xRMS}=1 / \mathrm{T}\left(\int_{0}^{T} x^{2} d t\right) 1 / 2$

This equation identifies with the assessment of vibration in time and the vitality substance of the vibration wave. For the unitary sufficiency sine wave, the RMS esteem is 0.707 of the pinnacle adequacy. The least complex case is the free vibration of a molecule without damping.

\section{Introduction to Motion Magnification}

Euler video amplification is a method used to bring little developments caught up in video. A few potential applications incorporate estimation of auxiliary vibration. Since a video normally catches the whole scene at the same time, it is normally appropriate for the investigation of modular vibration, where the stage connection between various spatial areas is significant.

(Chen et al., 2015) utilized video intensification to gauge 1D mode shapes on the cantilever bar and the cross area of a roundabout cylinder. Particularly vacuum cleaners, hand dryers, cooling fans, engines, structures and so on. We expected to apply and ex tend this method to permit vibration investigation in mechanical clamor control designing for such items. Applying video amplification to the vibration estimation at that point requires breaking down complex geometries, having quantitative and subjective yield, and strong against clamor.

\section{About Test Environment}

In this study, the movements that are not visible or difficult to see with the naked eye can be seen in the videos. In another definition, it is to reveal the temporal variations in the videos that are difficult or impossible to see with the naked eye and make them visible. Many images around us contain movements that are too small to be detected by the naked eye. The world is filled with fine and small movements that cannot be seen with the naked eye. For example, human skin color changes slightly according to blood circulation. When the heart pumps blood into the body, there is an invisible color change in the veins just under our skin. Although this variation is not visible to the naked eye, it can be used to extract the pulse rate. Similarly, low spatial amplitude movements, although difficult or impossible for people to see, can be magnified and many comments can be made from these data to reveal interesting mechanical behavior.

Our basic approach is to examine the time series of color values in any spatial location (pixels) and the change in a specific temporal frequency band.

Our method, which we call SMART Video Magnification, takes standard video frames as input and applies the Spatial Decomposition Method, then applies temporary filtering to the frames. The resulting signal is then amplified to reveal confidential information. In this way, it is made visible by strengthening the color changes or small movements in the video. Our technique can work in real time to show events occurring at temporal frequencies selected by the user. In this study, a combination of spatial and temporal processing of videos has been shown to reveal important aspects of the world around us that are unseen. Our temporary filtering approach can increase color diversity as well as reveal low amplitude motion.

Our approach combines spatial and temporal processing to emphasize subtle temporal changes in a video. First we divide the video sequence into different spatial frequency bands. These bands can be magnified differently because (a) they may exhibit different signalto-noise ratios (b) they may contain spatial frequencies that are not covered by the linear approach used in our motion amplific ation system. A mathematical analysis explaining how temporal filtering interacts with spatial motion in videos is presented below.

First order movement:

To explain the relationship between temporal processing and motion magnification, we consider the simple state of the 1D signal in motion.

Let's show the image density at position $\mathrm{x}$ and time $\mathrm{t}$ with $\mathrm{i}(\mathrm{x}, \mathrm{t})$. If the intensity observed according to the displacement function is $\delta$ $(\mathrm{t})$, since the image passes the translational motion.

It can be expressed as $I(x, t)=f(x+\delta(t))$ and $I(x, 0)=f(x)$.

The purpose of motion magnification is to synthesize the signal. Our formula for amplification factor $\alpha$

$I^{\wedge}(x, t)=f(x+(1+\alpha) \delta(t))$ 
It shaped.

Assuming the image can be predicted by first-order Taylor series expansion, the image $t$

If we express with the first degree Taylor expansion with respect to $\mathrm{x}$ in $\mathrm{f}(\mathrm{x}+\delta(\mathrm{t}))$ time

$\mathrm{I}(\mathrm{x}, \mathrm{t}) \approx \mathrm{f}(\mathrm{x})+\delta(\mathrm{t}) \partial \mathrm{f}(\mathrm{x}) \partial \mathrm{x}$.

For now, let's assume that the motion signal $\delta(\mathrm{t})$ is inside the temporary bandpass filter, let's say the result of applying a temporary band filter to $I(x, t)$ at each position of $B(x, t) x$. So,

$\mathrm{B}(\mathrm{x}, \mathrm{t})=\delta(\mathrm{t}) \partial \mathrm{f}(\mathrm{x}) / \partial \mathrm{x}$.

In our process, we reinforce this band pass signal with $\alpha$ and add it back to $\mathrm{I}(\mathrm{x}, \mathrm{t})$,

Our formula is

$I^{\sim}(x, t)=I(x, t)+\alpha B(x, t)$

When equations are combined 2,3,4

$\mathrm{I}^{\sim}(\mathrm{x}, \mathrm{t}) \approx \mathrm{f}(\mathrm{x})+(1+\alpha) \delta(\mathrm{t}) \partial \mathrm{f}(\mathrm{x}) / \partial \mathrm{x}$

When the equation 12 is reinterpreted, the following formula is reached

$\mathrm{I}^{\sim}(\mathrm{x}, \mathrm{t}) \approx \mathrm{f}(\mathrm{x}+(1+\alpha) \delta(\mathrm{t}))$

This formula shows us that the displacement of $\delta(t)$ at time $t$ on image $f(x)$ will increase by $(1+\alpha)$. Thus, the newly formed image function will also contain invisible movements. The details of the model developed in the figure below are given. Fig. 1.5

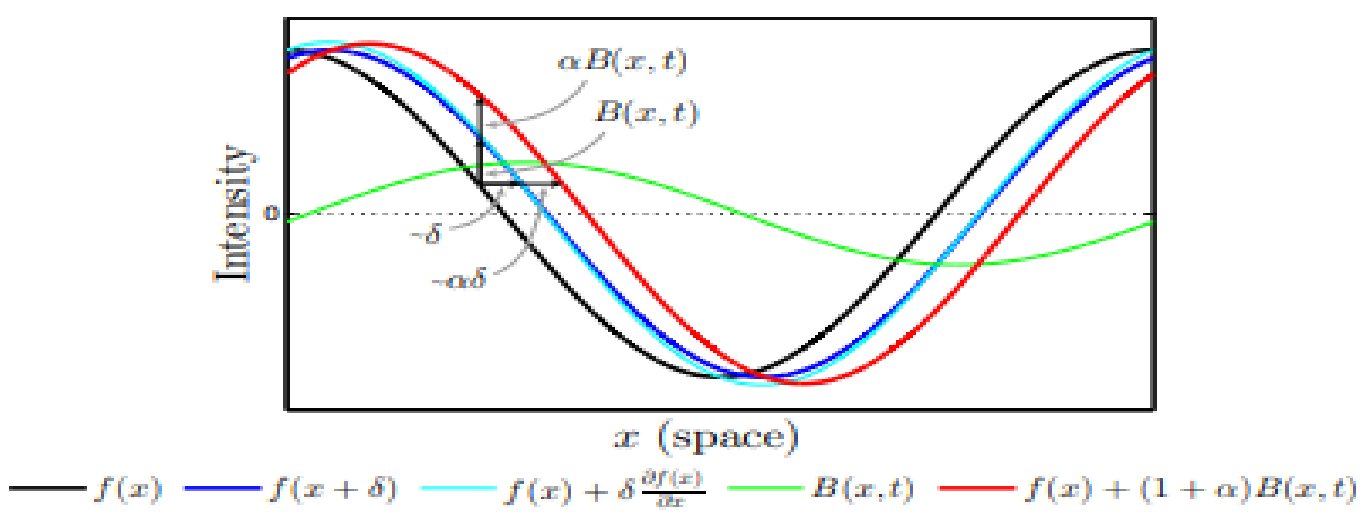

Fig. 5. The details of the model developed

Movements that are not visible to the naked eye or difficult to see are provided. In this study, the mathematical modeling mentioned above was coded and successfully operated. The system takes the video images as input, then separates them into fraems and applies a $5 * 5$ filter on it and derives its time by time. The resulting images are added to the original image with the $\alpha$ coefficient, as expressed in equation 13 .

\section{Test Equipment}

The hardware developed within the scope of this project and related to the motion magnification analysis software were used together. With the image acquired by the camera taken from Basler, the project output was provided to be run on various videos we obtained from our mobile phones. In addition, videos of industrial machines were shot at various locations and software was tried to ensure that they were working properly in field conditions. In these videos, video magnification was possible. Thus, the software has been verified and successfully executed by comparing both the videos in the academic literature and the videos we shot.

The "Test and Calibration Procedures" work package is the sixth work package. In this package, it is tried to compare the accuracy and consistency of the inferences made from the images taken using the mechanical vibration measuring device purchased in the first work package. For this purpose, we have taken videos for testing purposes in areas such as MKE Arms Factory, DDF Construction Basalt Quarry and Doğuş Construction Ankara-Sivas High Speed Train Line Construction sites and studies have been done. The purpose here is to test and calibrate the software, as mentioned in the work package, and it has been realized. In this section, the procedures for obtaining frequencies for various videos are made from the screenshots taken from the software. The following equipment has been used for testing and verification. Fig.6, Fig.7, Fig.8. 


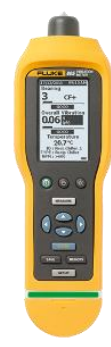

Fig. 6. Fluke 805 FC Vibration Meter

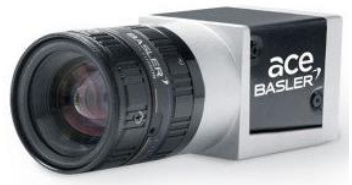

Fig. 7. High Speed Camera

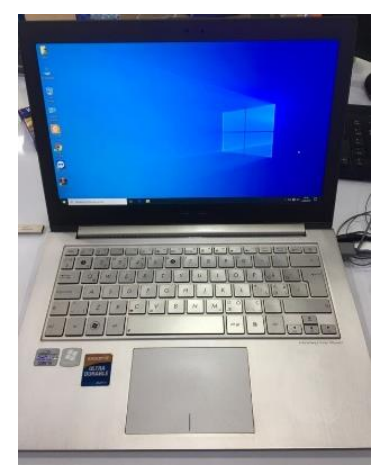

Fig. 8. C\# and .NET Enviroment Computer

\section{Test Result Samples}

In this section, it is tried to compare the accuracy and consistency of the inferences made from the images taken by using the purchased mechanical vibration measuring device. For this purpose, we have taken videos for testing purposes in areas such as MKE Arms Factory, DDF Construction Basalt Quarry and Doğuş Construction Ankara-Sivas High Speed Train Line Construction sites and studies have been done. The purpose here is to test and calibrate the software as it is said in the work package and it has been realized. In this section, the procedures for obtaining frequencies for various videos are made from the screenshots taken from the software and the results are given in the pictures below.

However, as many different situations can be encountered in real life, the calibration part will continue to be developed. Therefore, it is necessary to calibrate the software with benchmarking. Using the "Fluke 805 FC Vibration Meter" which bought from the project for this purpose, the application was made on the vehicle shown in Figure 9. Thus, the software has been calibrated.
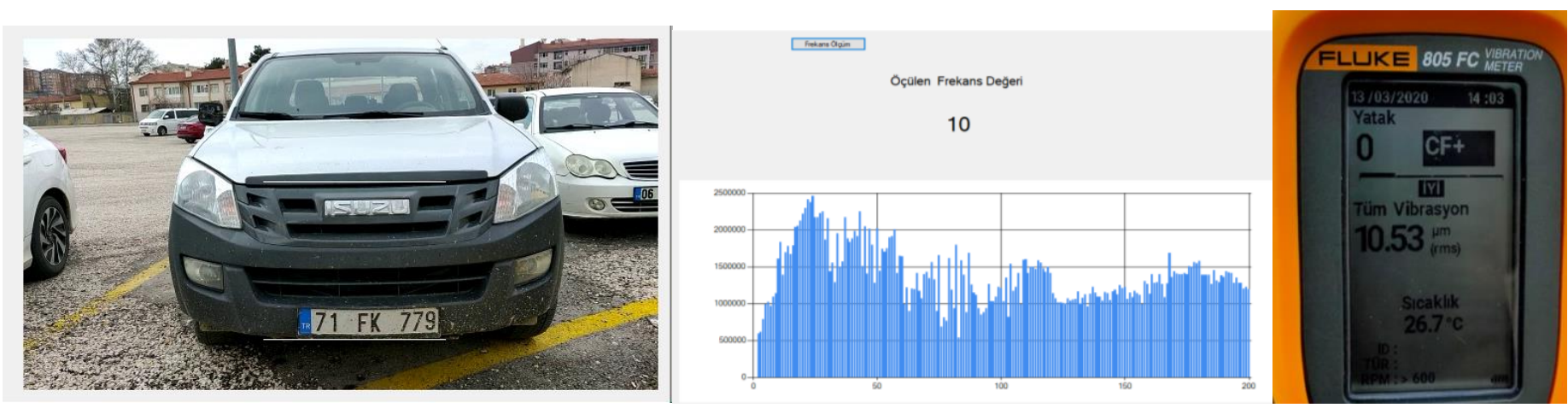

Fig. 9. Calibration of the software with Benchmarking 


\section{Conclusions And Future Work}

This article is about a system designed to predict the failures that industrial devices and/or various structures can cause due to wear of time. The idea of changing the frequency of vibrations of the aging devices while working caused this. All these applications were supported by a KOSGEB project by made the AES Innovaion Inc. in Turkey. For this purpose, a combined hardware and software has been designed. In order to show the accuracy of the results and calibrate the system, comparative applications were made with the "Fluke 805 FC Vibration Meter" device. In the study, it has been demonstrated that rapid video intensification is applied to vibration estimation. Video amplification procedure (Correa \& Guzman, 2020) and application to modular investigation (Chen et a have been recently portrayed in the writing. There are likewise more current investigations of handling rapid video to gauge vibration (Mas et al., 2016). As to past work, the development in this article initially portrayed a viewpoint revision that permitted the quantitative estimation of 2D alterations, an expansion in the estimation of 1D mode shapes, and a progressively subjective examination detailed in different investigations. Second, a video examination utilizing sifting has been appeared to precisely extricate mode designs when the structure is invigorated utilizing commotion and cleared sine signals, once more, as far as anyone is concerned, this has not been recently announced. At last, it has been shown an exact application for designing vibration investigation utilizing an operational item.

In the future, the subsequent stage for this investigation is to stretch out the point of view revision to complex surfaces, as we limit our examination to level plates. Estimating 3D vibration of complex shapes may require a connection between's various camera areas. Another region of research is to utilize an information power converter to compute emanated acoustic weight or to utilize vibration information dependent on radiation impedance or to ascertain structure versatility. Within the scope of the project, it is aimed to produce integrated solutions with thermal camera integration in addition to vibration analysis of buildings and machines. In addition, as a result of the literature searches, it was seen that the technique we developed as a biomedical diagnostic method can be used(Li \& Lin, 2017), (Monkaresi et al., 2016), (Hassan et al., 2017).

\section{Acknowledgements}

This project was supported by KOSGEB (2019/1695597) (Small and Medium Enterprises Development Organization of Turkey). We would like to thank KOSGEB and Kırıkkale University Technopark Management for their contribution.

\section{References}

Ashory, M. R. (1999). High quality modal testing methods (Doctoral dissertation, University of London).

Chen, J. G., Wadhwa, N., Cha, Y. J., Durand, F., Freeman, W. T., \& Buyukozturk, O. (2015). Modal identification of simple structures with high-speed video using motion magnification. Journal of Sound and Vibration, 345, 58-71.

Correa, J. C. A. J., \& Guzman, A. A. L. (2020). Mechanical Vibrations and Condition Monitoring. Elsevier Science \& Technology.

Davis, A., Rubinstein, M., Wadhwa, N., Mysore, G. J., Durand, F., \& Freeman, W. T. (2014). The visual microphone: Passive recovery of sound from video

Gonzales, R. (1987). Wintz, Paul. Digital Image Processing.

Hassan, M. A., Malik, A. S., Fofi, D., Saad, N. M., Ali, Y. S., \& Meriaudeau, F. (2017). Video-based heartbeat rate measuring method using ballistocardiography. IEEE Sensors Journal, 17(14), 4544-4557.

Li, M., \& Lin, J. (2017). Wavelet-transform-based data-length-variation technique for fast heart rate detection using 5.8-GHz CW Doppler radar. IEEE Transactions on Microwave Theory and Techniques, 66(1), 568-576.

Mas, D., Ferrer, B., Acevedo, P., \& Espinosa, J. (2016). Methods and algorithms for video-based multi-point frequency measuring and mapping. Measurement, 85, 164-174.

Monkaresi, H., Bosch, N., Calvo, R. A., \& D'Mello, S. K. (2016). Automated detection of engagement using video-based estimation of facial expressions and heart rate. IEEE Transactions on Affective Computing, 8(1), 15-28.

Wadhwa, N., Rubinstein, M., Durand, F., \& Freeman, W. T. (2013). Phase-based video motion processing. ACM Transactions on Graphics (TOG), 32(4), 1-10. 